\title{
BMJ Open Interventions for Menière's disease: protocol for an umbrella systematic review and a network meta-analysis
}

\author{
Babette F van Esch, ${ }^{1}$ Hester J van der Zaag-Loonen, ${ }^{1}$ Tjasse D Bruintjes, ${ }^{1}$ \\ Peter Paul G van Benthem ${ }^{2}$
}

To cite: van Esch BF, van der Zaag-Loonen HJ, Bruintjes TD, et al. Interventions for Menière's disease: protocol for an umbrella systematic review and a network meta-analysis. BMJ Open 2016;6:e010269. doi:10.1136/bmjopen-2015010269

- Prepublication history for this paper is available online To view these files please visit the journal online (http://dx.doi.org/10.1136/ bmjopen-2015-010269).

Received 15 October 2015 Revised 26 April 2016 Accepted 6 May 2016

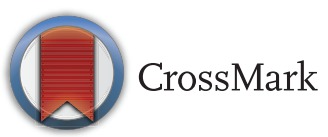

${ }^{1}$ Apeldoorn Dizziness Centre, Gelre Hospital, Apeldoorn, The Netherlands

${ }^{2}$ Leiden University Medical Centre, Leiden, The Netherlands

Correspondence to Babette $F$ van Esch; B.F. vanEsch@umcutrecht.nl

\section{ABSTRACT}

Introduction: The large number of treatment modalities for patients diagnosed with Menière's disease (MD) complicates the selection of the best available treatment as the comparative efficacy of these interventions is not clear. We aim to identify the treatment or treatments with the highest efficacy of current pharmacological and non-pharmacological treatments for MD.

Methods and analysis: We will identify all available systematic reviews on the treatment of MD. An online database search will be conducted in association with the UK Cochrane Centre, particularly the Ear, Nose and Throat Group. We will screen the systematic reviews for eligible randomised controlled trials (RCTs) to execute a network meta-analysis. In addition, online databases will be checked for eligible RCTs on treatments that were published after the latest systematic search was conducted. The characteristics of each RCT will be summarised, including the general design, the participants, the interventions, the outcome measurements, the duration of therapy and adverse events. The risk of bias will be assessed by means of the Cochrane Collaboration's risk of bias tool. The included studies will be assessed for methodological and statistical heterogeneity; the latter will be quantified by means of the $I^{2}$ statistic. The primary outcome will be the efficacy of treatment in terms of control of vertigo attacks. Secondary outcome measures will be the loss or improvement of hearing, severity of vertigo attacks and tinnitus, perception of aural fullness, quality of life, and the incidence of adverse events and complications.

Ethics and dissemination: Formal ethical approval is not required as primary data will not be collected. The review will be disseminated in peer-reviewed publications and conference presentations.

\section{PROSPERO registration number:} CRD42015024243.

\section{INTRODUCTION}

Menière's disease (MD) is an inner ear disorder characterised by incapacitating attacks of vertigo accompanied by nausea and vomiting, fluctuating sensorineural hearing loss as

\section{Strengths and limitations of this study}

Until now, this is the first umbrella systematic review protocol to summarise the evidence for pharmacological and non-pharmacological treatments for Menière's disease.

- The protocol has been created according to the published PRISMA-P guidelines.

- We aim to identify the treatment or treatments with the highest efficacy for Menière's disease based on controlling vertigo or reducing the frequency of vertigo attacks.

- The main limitation of this study identifying all the interventions for Menière's disease will be overcome by seeking all interventional systematic reviews that have been published and adding randomised controlled trials that were published subsequent to the date the latest systematic search was conducted.

well as tinnitus and/or aural fullness. Even though the disease was first described in 1861 by Menière, ${ }^{1}$ there are still many unanswered questions regarding the pathophysiology of the disease. Furthermore, a definite and effective evidence-based treatment has not been established yet.

The main aim of the treatment in MD is to reduce the frequency and intensity of the vertigo attacks and, at the same time, to preserve hearing and vestibular function. ${ }^{2}$ Psychological suffering and reduced quality of life are linked to $\mathrm{MD}$, as disabling vertigo attacks can occur without warning. ${ }^{3}{ }_{4}$ Therefore, an effective prophylactic treatment is necessary to improve the quality of life of patients with MD. Current pharmacological treatment options include betahistine, diuretics, oral steroids or intratympanic application of gentamicin or corticosteroids. ${ }^{5}$ However, evidence in terms of reducing vertigo symptoms has never been conclusive ${ }^{6-8}$ except for intratympanic gentamicin treatment. $^{9}$ Non-pharmacological treatment options include positive pressure therapy 
(the Meniett device), ablative surgery such as vestibular nerve section, labyrinthectomy and endolymphatic sac surgery. ${ }^{2} 510$ As for the pharmacological treatment modalities, high-quality evidence is also lacking for nonpharmacological therapies. ${ }^{10} 11$ Since so many treatments exist without conclusive results, it may be hard for patients and their physicians to select the best available treatment. Until now, no umbrella systematic review (SR) exists that summarises the body of evidence and states implications for clinical practice.

\section{Objective}

The present study aims to systematically summarise the interventions for $\mathrm{MD}$, aiming to identify the treatment or treatments with the highest efficacy and to identify areas for future valuable research.

\section{METHODS}

Study design

A large number of pharmacological and nonpharmacological trials for the treatment of MD exist. We will conduct an umbrella SR of published randomised controlled trials (RCTs) of those interventions that have been systematically reviewed. From here we seek to evaluate the efficacy of therapy for MD. The current review has been registered at PROSPERO CRD42015024243. The steps throughout the conduct of the umbrella SR are shown in figure 1 . This protocol is reported in line with PRISMA-P. ${ }^{12}$

\section{Eligibility criteria}

Types of studies

The following study designs will be eligible for inclusion:

- SR or meta-analysis (MA),

- RCTs or placebo controlled trials.

We will screen interventional SRs for eligible RCTs and data from these RCTs will be extracted to execute a network MA. In addition, online databases will be checked for eligible RCTs on treatments that were systematically reviewed yet published subsequent to the date the latest systematic search was conducted.

\section{Types of participants}

Owing to the great variability in the clinical presentation of MD, the disorder is not always easy to diagnose. The American Academy of Otolaryngology-Head and Neck Surgery (AAO-HNS) has produced diagnostic guidelines in order to facilitate the diagnosis of MD and to improve comparability of outcome measures when performing trials on patients with MD. ${ }^{13}$ In 2015, a new set of diagnostic criteria were jointly formulated by the Classification Committee of the Bárány Society, the Japan Society of Equilibrium Research, the European Academy of Otology and Neurotology, the AAO-HNS and the Korean Balance Society in order to develop an international consensus on diagnostic criteria for MD in order to facilitate future collaborative studies. ${ }^{14}$ However,
Electronic database search for systematic reviews of treatment in Menière's disease

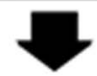

Screening for systematic reviews and RCTs published after conduction of the systematic search

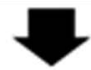

Include eligible RCTs from systematic reviews
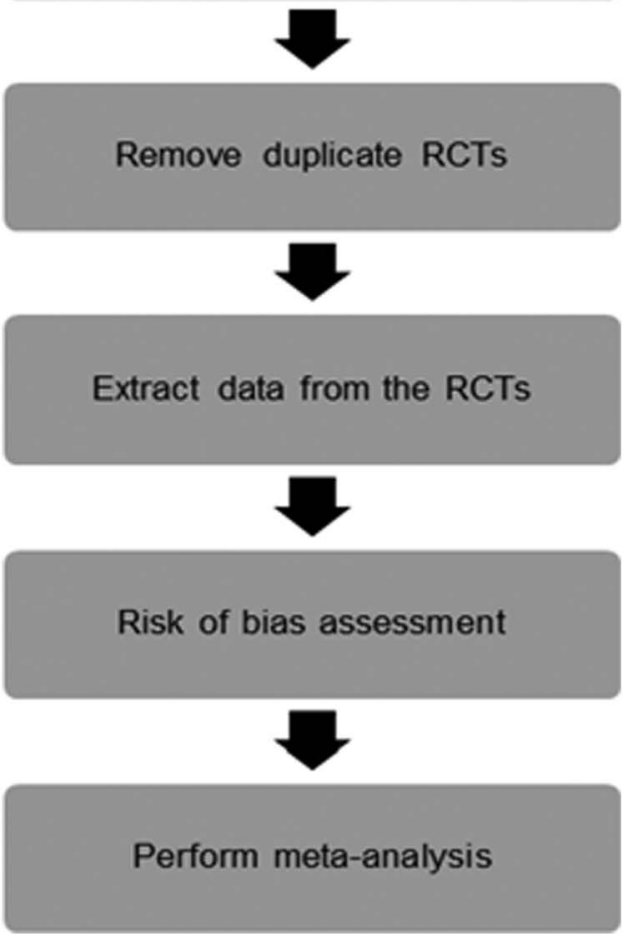

Figure 1 Flow chart of the umbrella systematic review. RCT, randomised controlled trial.

since these international diagnostic criteria were only published recently and previous research widely used the AAO-HNS 1995 diagnostic guidelines, the latter set of criteria will be used to identify patients with 'definite' MD in the current review.

\section{Types of intervention}

We will include RCTs analysing the efficacy of any treatment modality in MD. Treatment modalities that have not been assessed systematically will not be included in the umbrella SR. Since the natural course of MD has a waning pattern, time should be regarded as a therapeutic factor when analysing the efficacy of a therapeutic intervention. Therefore, a study design including a placebo arm is essential to account for the illusion of therapeutic efficacy. Pharmacological trials with a 
placebo group will be included; trials comparing different pharmacological treatments without a placebo will be excluded. We will include trials that investigated nonpharmacological interventions and compared the efficacy of the intervention with a sham intervention group, a placebo pill group or a placebo control group.

\section{Types of outcome measures}

Outcomes as defined by the AAO-HNS guidelines of $1995^{13}$ will be included in this umbrella SR. The following outcomes are listed as primary and secondary outcomes:

\section{Primary outcomes}

1. The main outcome of efficacy will be the control of vertigo as defined by the AAO-HNS guidelines of $1995 .{ }^{13}$ The number of vertigo attacks in the interval after treatment $(\mathrm{Y})$ is divided by the number of vertigo spells 6 months prior to treatment $(\mathrm{X})$ and multiplied by 100 . The resulting number indicates the extent of 'control of vertigo'. The AAO-HNS further divides the control of vertigo into classes, where class $\mathrm{A}(\mathrm{CoV}=0)$ represents a complete control of vertigo and class B (CoV up to 40\%) represents a substantial control of vertigo. Assessment of control of vertigo by any other outcome measures (eg, mean frequency of vertigo attacks at baseline and at the final assessment) will also be accepted.

\section{Secondary outcomes}

Secondary outcome measures will be:

1. Hearing (based on pure-tone audiometry).

2. The severity of vertigo attacks (assessed by means of a standardised method (eg, the visual analogue scale (VAS) or the MD Patients Oriented Severity Index (MD-POSI) ).

3. The severity of tinnitus (assessed by means of a standardised method (eg, VAS, Tinnitus Handicap Inventory)).

4. Perception of aural fullness (assessed by means of a standardised method (eg, VAS)

5. Quality of life (generic quality of life (eg, SF-36) and/or disease-specific quality of life (eg, Functional Level Scale, Dizziness Handicap Index)).

6. The incidence of adverse events or complications.

\section{Search strategy}

In association with the UK Cochrane Centre, particularly the Ear, Nose and Throat Review Group, we will conduct a systematic search for all SRs for pharmacological and non-pharmacological interventions for MD. We will search the Database of Abstracts of Reviews of Effect (DARE), MEDLINE and EMBASE for SRs, and eligible RCTs will be extracted that examine the efficacy of pharmacological and non-pharmacological therapies in MD. In case several SRs investigate exactly the same treatment modality in the same population, we will extract the RCTs from the most recent published review.
Table 1 Search strategy for systematic reviews for Menière's disease
\#1

\#2

\#3

\#4

\#6

\#7 systematic review \#1 AND \#2 meta-analysis \#1 AND (\#3 OR \#4) \#3 OR \#6 exp Meniere disease* [therapy]

Since no current worldwide-recommended guidelines exist for the treatment of MD, we intend to include all systematically reviewed interventions. We will use Medical Subject Headings (MeSH) and key words in the search strategy containing 'Menière's disease', 'systematic review', 'randomised controlled trial' and 'placebo controlled trial'. Details of the search strategy are shown in tables 1 and 2.

Two independent reviewers (BFvE and HJvdZ-L) will screen the title and abstract for potentially eligible SRs. These will be downloaded for full-text screening and further evaluation. Authors and journal names will be blinded. No restriction on language will be used. After identifying all interventions that were systematically reviewed, we will screen the title and abstract for potentially eligible RCTs that were published since the publications of these SRs. Similar to the selection of SRs, these RCTs will be screened on full text and evaluated. We will remove all duplicate RCTs after full-text screening and reference checking. The reviewers will examine and extract all data from the included RCTs into a data set.

\section{Data extraction}

After we selected eligible RCTs, the two reviewers (BFvE and HJvdZ-L) will independently extract information from the RCTs on predesigned data extraction forms. To begin with, we will extract the general information from each RCT covering the country, number of centres, number of participants, study design, the number of treatment arms, allocation ratio, and conflict of interest and funding. Then the study characteristics of the patients with MD will be extracted including sex, age, age at onset of disease, subclassification of MD types (diagnostic criteria defined by the AAO-HNS of 1995) and duration and frequency of vertigo attacks before start of treatment. Furthermore, details of the interventions will be extracted for the experimental and control

Table 2 Search strategy for randomised controlled trials for Menière's disease
$\# 1$

$\# 2$

$\# 3$

\#4

\#6

$\# 7$ randomised controlled trial \#1 AND \#2 placebo controlled trial \#1 AND (\#3 OR \#4) \#3 OR \#6 exp Meniere disease* [therapy] 
groups. For the pharmacological interventions, we will record the drug category (eg, anticholamines, diuretics), generic name of the drug, dose per day, way of administration (eg, oral, intratympanic), additional treatments and period of treatment. In addition, for the nonpharmacological interventions, we will extract the type of intervention (eg, Meniett device, endolymphatic sac surgery) and any additional treatments (prestudy or during trial participation). Lastly, we will extract information of the effect on the primary and secondary outcome measures and record the incidence of adverse events and complications. We will extract the information from the intervention arm in pharmacological and non-pharmacological RCTs using a standard form (tables 3 and 4). In addition, tables 5 and 6 display the standard form that will be used to extract information from the placebo arm in pharmacological RCTs and the sham arm in non-pharmacological RCTs, respectively.

\section{Outcome assessment}

We aim to investigate the efficacy of treatment for MD in controlling vertigo attacks (primary outcome). As defined in the AAO-HNS guideline of $1995,{ }^{13}$ the control of vertigo will be calculated and classified (class A, $100 \%$ control of vertigo, class B, $40 \%$ control of vertigo). Ideally, the primary outcome is again evaluated after 18 and 24 months following randomisation. However, it is unlikely that a placebo-controlled trial will last this long. Therefore, we will include papers that have assessed the efficacy of the therapy reflected by the primary outcome at 3-6 months of follow-up. We will ensure accurate assessment of the outcome measures as independent reviewers (BFvE and HJvdZ-L) extract the information from the selected RCTs and a third reviewer (TDB and/or PPGvB) will check the completeness and correctness of the extracted data.

\section{Risk of bias assessment}

We will assess the methodological quality of the RCTs by use of the Cochrane Collaboration's risk of bias tool ${ }^{14}$ within Review Manager V.5.3 software (Review Manager (Revman) V.5.3 Copenhagen: The Nordic Cochrane Centre, The Cochrane Collaboration, 2012). The tool is based on the following eight potential sources of bias: random sequence generation; allocation concealment; blinding of the participants; blinding of the outcome assessors; incomplete outcome data; missing data and selective outcome reporting, other bias (eg, improper statistical analysis). Two independent reviewers (BFvE and HJvdZ-L) will independently evaluate the quality of the RCTs. Each aspect will be graded with 'yes', 'no' or 'unclear', which will reflect a high risk of bias, low risk of bias and unclear risk of bias, respectively. For each study, all eight domains will be evaluated and displayed in a table (see table 7). If there is any disagreement on inclusion or exclusion, this will be settled by discussion, if necessary in the presence of a third reviewer (TDB and/or PPGvB). In addition, we will grade the

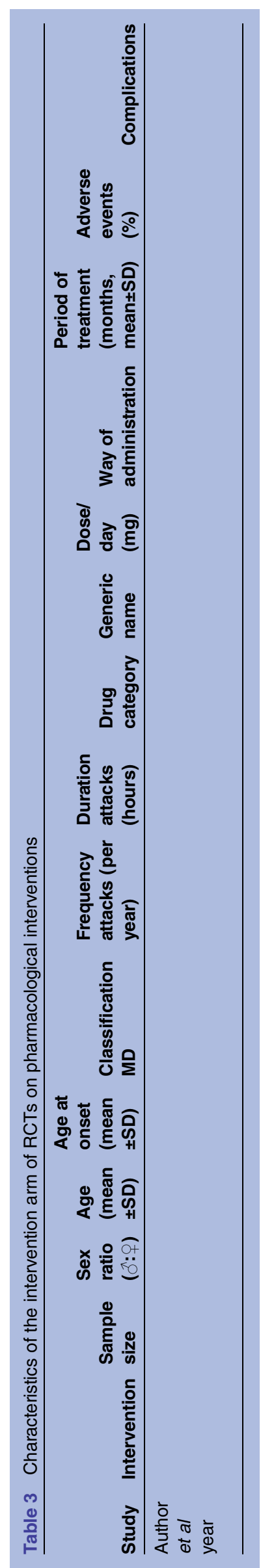


Table 4 Characteristics of the intervention arm of RCTs on non-pharmacological interventions

\begin{tabular}{|c|c|c|c|c|c|c|c|c|c|c|c|c|}
\hline Study & Intervention & $\begin{array}{l}\text { Sample } \\
\text { size }\end{array}$ & $\begin{array}{l}\text { Sex } \\
\text { ratio } \\
\left({ }^{\prime}: P\right)\end{array}$ & $\begin{array}{l}\text { Age } \\
\text { (mean } \\
\pm S D \text { ) }\end{array}$ & $\begin{array}{l}\text { Age at } \\
\text { onset } \\
\text { (mean } \\
\pm \text { SD) }\end{array}$ & $\begin{array}{l}\text { Classification } \\
\text { MD }\end{array}$ & $\begin{array}{l}\text { Frequency } \\
\text { attacks (per } \\
\text { year) }\end{array}$ & $\begin{array}{l}\text { Duration } \\
\text { attacks } \\
\text { (hours) }\end{array}$ & $\begin{array}{l}\text { Additional } \\
\text { treatment }\end{array}$ & $\begin{array}{l}\text { Period of } \\
\text { treatment } \\
\text { (months, } \\
\text { mean } \pm S D \text { ) }\end{array}$ & $\begin{array}{l}\text { Adverse } \\
\text { events } \\
\text { (\%) }\end{array}$ & Complications \\
\hline $\begin{array}{l}\text { Author } \\
\text { et al } \\
\text { year }\end{array}$ & & & & & & & & & & & & \\
\hline
\end{tabular}

Table 5 Characteristics of the placebo arm of RCTs on pharmacological interventions

\begin{tabular}{|c|c|c|c|c|c|c|c|c|c|c|c|c|c|c|}
\hline Study & Intervention & $\begin{array}{l}\text { Sample } \\
\text { size }\end{array}$ & $\begin{array}{l}\text { Sex } \\
\text { ratio } \\
\left(\delta^{1}: 9\right)\end{array}$ & $\begin{array}{l}\begin{array}{l}\text { Age } \\
\text { (mean }\end{array} \\
\pm \text { SD) } \\
\end{array}$ & $\begin{array}{l}\text { Age at } \\
\text { onset } \\
\text { (mean } \\
\pm \text { SD) } \\
\end{array}$ & $\begin{array}{l}\text { Classification } \\
\text { MD }\end{array}$ & $\begin{array}{l}\text { Frequency } \\
\text { attacks (per } \\
\text { year) }\end{array}$ & $\begin{array}{l}\text { Duration } \\
\text { attacks } \\
\text { (hours) }\end{array}$ & $\begin{array}{l}\text { Drug } \\
\text { category }\end{array}$ & $\begin{array}{l}\text { Dose/ } \\
\text { day } \\
\text { (mg) }\end{array}$ & $\begin{array}{l}\text { Way of } \\
\text { administration }\end{array}$ & $\begin{array}{l}\text { Period of } \\
\text { treatment } \\
\text { (months, } \\
\text { mean } \pm S D)\end{array}$ & $\begin{array}{l}\text { Adverse } \\
\text { events } \\
(\%)\end{array}$ & Complications \\
\hline $\begin{array}{l}\text { Author } \\
\text { et al } \\
\text { year }\end{array}$ & & & & & & & & & & & & & & \\
\hline
\end{tabular}

Table 6 Characteristics of the sham arm of RCTs on non-pharmacological interventions

\begin{tabular}{|c|c|c|c|c|c|c|c|c|c|c|c|c|}
\hline Study & $\begin{array}{l}\text { Sham } \\
\text { intervention }\end{array}$ & $\begin{array}{l}\text { Sample } \\
\text { size }\end{array}$ & $\begin{array}{l}\text { Sex } \\
\text { ratio } \\
(\delta: \uparrow)\end{array}$ & $\begin{array}{l}\text { Age } \\
\text { (mean } \\
\pm S D \text { ) }\end{array}$ & $\begin{array}{l}\text { Age at } \\
\text { onset } \\
\text { (mean } \\
\pm S D \text { ) }\end{array}$ & $\begin{array}{l}\text { Classification } \\
\text { MD }\end{array}$ & $\begin{array}{l}\text { Frequency } \\
\text { attacks (per } \\
\text { year) }\end{array}$ & $\begin{array}{l}\text { Duration } \\
\text { attacks } \\
\text { (hours) }\end{array}$ & $\begin{array}{l}\text { Additional } \\
\text { treatment }\end{array}$ & $\begin{array}{l}\text { Period of } \\
\text { treatment } \\
\text { (months, } \\
\text { mean } \pm S D \text { ) }\end{array}$ & $\begin{array}{l}\text { Adverse } \\
\text { events } \\
\text { (\%) }\end{array}$ & Complications \\
\hline $\begin{array}{l}\text { Author } \\
\text { et al } \\
\text { year }\end{array}$ & & & & & & & & & & & & \\
\hline
\end{tabular}




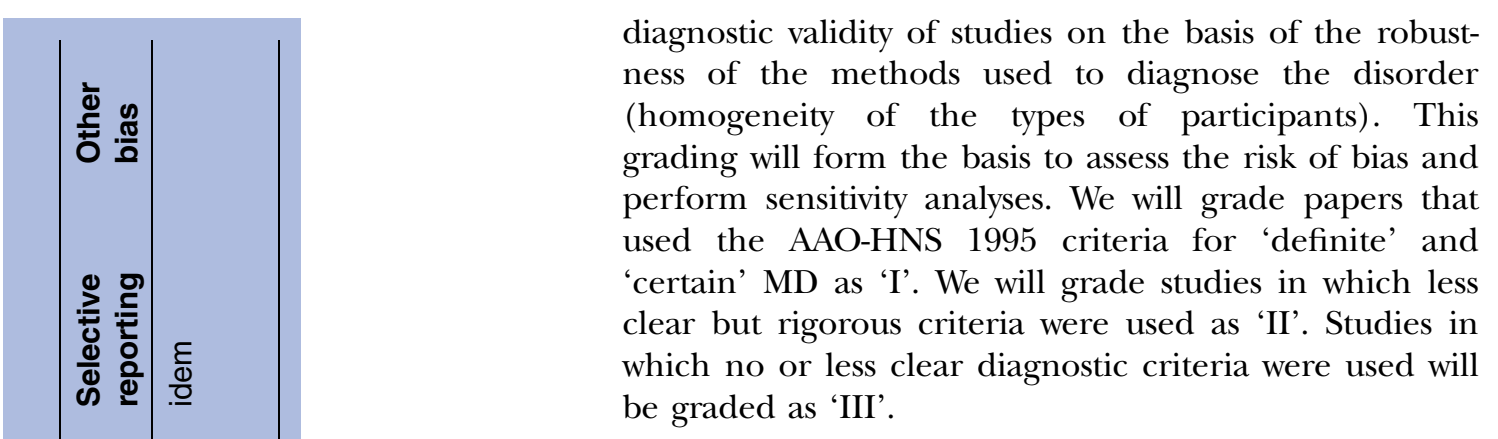

\section{Data analysis}

Data will be entered into Review Manager (V.5.3). For each treatment modality, we aim to perform a statistical analysis for the primary outcome comparing the interventional arm to the control group (placebo or sham). In addition, for studies that report the vertigo attack frequency as a continuous outcome, we intend to calculate the effect size using the mean difference (MD) or the standardised MD. The same applies for the loss of hearing. Where appropriate, data will be categorised or dichotomised for control of vertigo, severity of vertigo attacks, severity of tinnitus, perception of aural fullness, quality of life, complications and adverse events.

The included studies will be explored on methodological and statistical heterogeneity. The latter will be quantified by the $\mathrm{I}^{2}$ statistic. An $\mathrm{I}^{2}$ value $>50 \%$ is considered to indicate substantial heterogeneity (Handbook 2011, The Cochrane Collaboration). ${ }^{15}$ If the data are sufficiently homogeneous, we will pool outcome data. It is expected that the data will carry a certain amount of heterogeneity and a random-effects model will be used. Forest plots will be shown for each intervention. If the data turn out to be too heterogeneous for pooling based on methodological heterogeneity and statistical heterogeneity, we will perform a descriptive review and summarise the available evidence for this intervention. The strength of the evidence will be evaluated by use of the GRADE method as generated by the Cochrane Collaboration. Table 8 shows the summary of findings per intervention based on the GRADE method.

\section{Dealing with missing data}

We expect missing data in the selected trials for the SR. All corresponding authors will be contacted and asked for the original data. If only a per protocol analysis has been carried out, corresponding authors will be contacted for the original data on the intention-to-treat analysis.

\section{Subgroup analysis}

We will perform subgroup analysis to investigate heterogeneity and inconsistency in the selected trials. Subgroup analysis will be performed with regard to subtype of MD ('certain', 'definite', 'probable' or 'possible' MD in accordance with the AAO-HNS 1995 criteria ${ }^{13}$ ), stage of disease (as defined by the AAO-HNS 
Table 8 Summary of findings per intervention

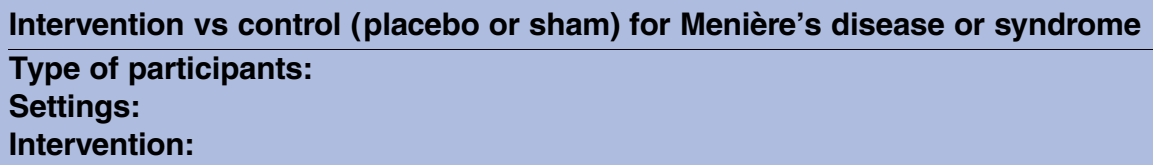

Outcomes

follow-up: mean...

\section{months}

We will use the GRADE approach to rate the overall quality of evidence. The quality of evidence reflects the extent to which we are confident that an estimate of effect is correct and we will apply this to the interpretation of results. There are four possible ratings: high, moderate, low and very low. A rating of high quality of evidence implies that we are confident in our estimate of effect and that further research is very unlikely to change our confidence in the estimate of effect. A rating of very low quality implies that any estimate of effect obtained is very uncertain. The GRADE approach rates evidence from RCTs that do not have serious limitations as high quality. However, several factors can lead to the downgrading of the evidence to moderate, low or very low. The degree of downgrading is determined by the seriousness of these factors: study limitations (risk of bias); inconsistency; indirectness of evidence; imprecision and publication bias.

This table will be constructed according to the recommendations described in Chapter 10 of the Cochrane Handbook for Systematic Reviews of Interventions (Handbook 2011). We will include the following outcomes in the 'Summary of findings' table: control of vertigo attacks, hearing, severity of vertigo attacks and tinnitus, perception of aural fullness, quality of life and adverse events.

*The basis for the assumed risk (eg, the median proportion of patients with control of vertigo related to the follow-up) will be provided in footnotes. The corresponding risk (and its $95 \% \mathrm{Cl}$ ) will be based on the assumed risk in the comparison group and the relative effect of the intervention (and its $95 \% \mathrm{Cl}$ ).

$\mathrm{RCT}$, randomised controlled trial.

1995 criteria $^{13}$ ) and duration of treatment. Since the primary outcome is a patient-reported outcome, blinding can be of influence. Therefore, we will consider the method of blinding the most important subgroup analysis.

\section{Sensitivity analysis}

We will perform a sensitivity analysis to address whether the eight potential sources of bias played a relevant role in the robustness of our study findings. Studies with a high risk of bias will be analysed separately to evaluate if the efficacy of the intervention is not solely based on these trials and if trial results are robust.

\section{Publication bias}

Publication bias will be explored by performing funnel plots if sufficient data are available (10 or more studies).

\section{ETHICS AND DISSEMINATION}

Formal ethical approval is not required as primary data will not be collected. The findings will be disseminated in peer-reviewed journals and conference presentations.

\section{CONCLUSION}

We expect this umbrella SR to provide a systematic summary of evidence and aim to identify the treatment(s) with the highest efficacy for MD and areas for future valuable research.

Acknowledgements The authors are grateful for the support of the members of the ENT Group of the UK Cochrane Centre, particularly Jenny Bellorini and
Samantha Faulkner, for conducting the systematic searches for randomised controlled trials for interventions of Menière's disease.

Contributors BFvE, HJvdZ-L, TDB and PPGvB contributed to the design and conception of the study protocol. The search strategy was developed and run by the ENT Group. BFvE and HJvdZ-L will screen studies on the title and abstract and full text. If disagreement over inclusion or exclusion arises, this will be settled by discussion with all authors (BFvE, HJvdZ-L, TDB and/or $P P G v B)$. BFvE and HJvdZ-L will independently extract data from the articles and a third reviewer (TDB and/or PPGvB) will check the completeness and correctness of the extracted data of the outcome assessment. All authors drafted and revised this study protocol and approved it for publication.

Funding This work was supported solely from institutional and/or departmental sources from the Apeldoorn Dizziness Centre, Gelre Hospital, Albert Schweitzerlaan 31, 7334 DZ Apeldoorn, The Netherlands.

\section{Competing interests None declared.}

Provenance and peer review Not commissioned; externally peer reviewed.

Open Access This is an Open Access article distributed in accordance with the Creative Commons Attribution Non Commercial (CC BY-NC 4.0) license, which permits others to distribute, remix, adapt, build upon this work noncommercially, and license their derivative works on different terms, provided the original work is properly cited and the use is non-commercial. See: http:// creativecommons.org/licenses/by-nc/4.0/

\section{REFERENCES}

1. Menière $P$. Memoire sur les lesions de l'orellei interne dinnant lieu à des symptômes de congestion cerebrale apolectiforme. Gazette Med Paris 1861;16:597-601.

2. Minor LB, Schessel DA, Carey JP. Ménière's disease. Curr Opin Neurol 2004;17:9-16.

3. Best C, Eckhardt-Henn A, Tschan R, et al. Psychiatric morbidity and comorbidity in different vestibular vertigo syndromes. Results of a prospective longitudinal study over one year. J Neurol 2009;256:58-65.

4. Yardley L, Kirby S. Evaluation of booklet-based self-management of symptoms in Ménière's disease: a randomized controlled trial. Psychosom Med 2006;68:762-9. 
5. Sajjadi H, Paparella MM. Ménière's disease. Lancet 2008;372:406-14.

6. James AL, Burton MJ. Betahistine for Ménière's disease or syndrome. Cochrane Databsase Syst Rev 2001;(1):CD001873.

7. Thirlwall AS, Kundu S. Diuretics for Ménière's disease or syndrome. Cochrane Database Syst Rev 2006;(3):CD003599.

8. Phillips JS, Westerberg B. Intratympanic steroids for Ménière's disease or syndrome. Cochrane Database Syst Rev 2011;(7): CD008514.

9. Pullens B, van Benthem PP. Intratympanic gentamicin for Ménière's disease or syndrome. Cochrane Database Syst Rev 2011;(3): CD008234.

10. Pullens B, Verschuur HP, van Benthem PP. Surgery for Ménière's disease. Cochrane Database Syst Rev 2013;2: CD005395.
11. van Sonsbeek S, Pullens B, van Benthem PP. Positive pressure therapy for Ménière's disease or syndrome. Cochrane Database Syst Rev 2015;3:CD00008419.

12. Shamseer $\mathrm{L}$, Moher D, Clarke M, et al. Preferred reporting items for systematic review and meta-analysis protocols (PRISMA-P) 2015: elaboration and explanation. BMJ 2014;349:g7647.

13. Committee on Hearing and Equilibrium. Committee on Hearing and Equilibrium guidelines for the diagnosis and evaluation of therapy in Menière's disease. American Academy of Otolaryngology-Head and Neck Foundation. Inc. Otolaryngol Head Neck Surg 1995;113:181-5.

14. Lopez-Escamez JA, Carey J, Chung WH, et al. Diagnostic criteria for Menière's disease. J Vestib Res 2015;25:1-7.

15. Higgins JP, Thompson SG, Deek JJ, et al. Measuring inconsistency in meta-analyses. BMJ 2003;327:557-60. 